\title{
The impact of LncRNA dysregulation on clinicopathology and survival of pancreatic cancer: a systematic review and meta-analysis (PRISMA compliant)
}

Elahe Seyed Hosseini ${ }^{1,2}$, Ali Nikkhah ${ }^{3}$, Amir Sotudeh $^{3}$, Marziyeh Alizadeh Zarei ${ }^{2}$, Fatemeh Izadpanah ${ }^{4}$, Hossein Nikzad ${ }^{1,2}$ and Hamed Haddad Kashani ${ }^{1,2^{*}}$ (1)

\begin{abstract}
Purpose: An increasing number of studies have reported a significant association between long non-coding RNAs (IncRNAs) dysregulation and pancreatic cancers. In the present study, we aimed to gather articles to evaluate the prognostic value of long non coding RNA in pancreatic cancer.

Experimental design: We systematically searched all eligible articles from databases of PubMed, Web of Science, and Scopus to meta-analysis of published articles and screen association of multiple IncRNAs expression with clinicopathology and/or survival of pancreatic cancer. The pooled hazard ratios (HRs) and their $95 \%$ confidence intervals ( $95 \% \mathrm{Cls}$ ) were used to analysis of overall survival, disease-free survival and progression-free survival were measured with a fixed or random effects model.

Results: A total of 39 articles were included in the present meta-analysis. Our results showed that dysregulation of IncRNAs were linked to overall survival (39 studies, 4736 patients $\mathrm{HR}=0.41,95 \% \mathrm{Cl} 0.25 \pm 0.58$, random-effects in pancreatic cancer. Moreover, altered IncRNAs were also contributed to progression-free survival (8 studies, 1180 patients HR: 1.88, 95\% Cl (1.35-2.62) and disease-free survival (2 studies, 285 patients, HR: 6.07, 95\% Cl 1.28-28.78). In addition, our findings revealed the association between dysregulated RNAs and clinicopathological features in this type of cancer.
\end{abstract}

Conclusions: In conclusion, dysregulated IncRNAs could be served as promising biomarkers for diagnosis and prognosis of pancreatic cancer.

Keywords: Pancreatic cancer, LncRNAs, Overall survival, Disease-free survival, Progression-free survival, Clinicopathological features

*Correspondence: hamedir2010@gmail.com; haddadkashani-h@kaums.ac.ir ${ }^{2}$ Anatomical Sciences Research Center, Institute for Basic Sciences, Kashan University of Medical Sciences, Kashan, Iran

Full list of author information is available at the end of the article

\section{Introduction}

Pancreatic cancer (PC) is a worldwide challenging cancer characterized by poor prognosis, ranking as one of the most lethal human malignancy. The 5 -year overall survival (OS) of PC patients is less than $5 \%$, with median survival time between 3-6 months. However, progresses in early detection, surgical techniques and treatments strategies including chemotherapy and targeted therapy original author(s) and the source, provide a link to the Creative Commons licence, and indicate if changes were made. The images or other third party material in this article are included in the article's Creative Commons licence, unless indicated otherwise in a credit line to the material. If material is not included in the article's Creative Commons licence and your intended use is not permitted by statutory regulation or exceeds the permitted use, you will need to obtain permission directly from the copyright holder. To view a copy of this licence, visit http://creativecommons.org/licenses/by/4.0/. The Creative Commons Public Domain Dedication waiver (http://creativeco mmons.org/publicdomain/zero/1.0/) applies to the data made available in this article, unless otherwise stated in a credit line to the data. 
have resulted in better improvements in management of PC patients, dismal prognosis of the disease has not improved over years [1].

So, it is an urgent need to identify novel diagnostic and prognostic biomarkers associated with pancreatic cancer. In recently decades Long noncoding RNAs (lncRNAs) as type of RNA that do not encode proteins with a length of $>200 \mathrm{nt}$ and crucial role in several different biological processes in diverse human diseases such as development and progression of various cancers [2,3]. Also LncRNAs play a critical physiological role in apoptosis, metastasis, invasion, migration and cell proliferation in different cancers $[4,5]$. The dysregulation of different lncRNAs is reported to be potential prognostic indicators in multiple human cancers [6-9].

Previous meta-analysis has showed that high lncRNAs expression could be used as potential prognostic markers among Asian bladder cancer patients [10]. Also dysregulation of lncRNAs expression were significantly associated with clinicopathology and survival of breast cancer patients [11]. Also similar results have been reported in ovarian, cervical and prostate cancer [12-14]. In pancreatic cancer LncRNAs are identified in body fluids and are extensively found in the blood, saliva, urine, even pancreatic fluid and exosomes from tumors. And were done analysis about effect of potential of lncRNAs in the diagnosis and treatment of $\mathrm{PC}$ but it is not comprehensive and complete. Due to absence comprehensive article that summarize and conclude information in this field, in this study we systematically update analysis of related articles to confirm the potential prognostic value of lncRNAs in patients with pancreatic cancer. Furthermore, the association between IncRNAs and clinicopathological characteristics from published articles was investigated to update analysis rather than 2017 [15].

\section{Materials and methods}

This systematic review and meta-analysis was done based on the standard guidelines of Preferred Reporting Items for Systematic Reviews and Meta-analysis (PRISMA) (S1 Checklist) [16, 17].

\section{Search strategy}

A comprehensive literature search was performed by three independent reviewers (AS, AN, and ESH) through the PubMed, Web of Science, and Scopus for relevant papers published up to November 2020. The following search terms were used: (“"Long noncoding RNA"[tiab] OR "IncRNA"[tiab] OR "IncRNAs"[tiab] OR "lincRNA"[tiab] OR "lincRNAs"[tiab] OR "long non-coding RNA"[tiab] OR "long non protein coding RNA"[tiab] OR "RNA, Long Noncoding"[tiab] OR "Long intergenic non-coding RNA"[tiab]) AND ("Pancreatic tumor"[tiab] OR "Pancreatic cancer"[tiab] OR "Pancreatic neoplasm" [tiab] OR "Pancreatic carcinoma" [tiab] OR "Pancreatic malignancy"[tiab]) AND ("Prognosis"[tiab] OR "Prognostic"[tiab] OR "Predict"[tiab] OR "Survival"[tiab] OR "Overall survival"[tiab] OR "Survival rate"[tiab] OR "Outcome"[tiab] OR "Recurrence"[tiab])). Moreover, relevant articles were also reviewed manually in order to identify potentially eligible literature. No restrictions by the publication date or language were done.

\section{Inclusion and exclusion criteria}

Articles with the following criteria were included in the current meta-analysis: (1) original study conducted on human beings, (2) literature measured the relationship between expression level of lncRNAs with clinicopathological symptom and survival rate in patients with pancreatic cancer, (3) studies which reported sufficient data to estimate hazard ratios (HRs) and their 95\% confidence interval (95\% CI), and (4) literature published in English. Studies were excluded if they had the following criteria: (1) insufficient data for HR and 95\% CI estimation, (2) reviews, letters, laboratory articles and animal studies, (3) reported HRs for a combination of multiple lncRNAs. In addition, if a study had reported final results in different models, we included only the full-adjusted one.

\section{Data extraction}

Data extraction was done independently by three investigators to rule out any discrepancy. The following data were extracted from each study: (1) basic information including first author's name, year of publication and country of origin, (2) patients' characteristic information: ethnicity, study population, sample size and followup duration (3) lncRNA information: names of lncRNAs, expression status, detection methods, survival results, and cut-off definition, and analysis method for survival (4) relationship between expression level of lncRNAs and survival outcome or clinicopathological characteristics (5) HRs and their 95\% CIs for survival analysis. Any disagreements were resolved by discussion and consensus. The study quality was assessed via the Newcastle-Ottawa Scale (NOS) [18]. The NOS uses a star system ranging from 0 to 8 stars. Studies that achieved 7 or more stars were considered as high quality papers

\section{Statistical analysis}

HRs and 95\% CIs were obtained from studies or calculated from Kaplan-Meier survival curves using Engauge Digitizer version 4.1 [19] to calculated the overall pooled HR and 95\% CI for the association between lncRNAs and survival in PC. The pooled HR was calculated using fixed-effect model, or random-effect model in cases of 
high between-study heterogeneity. Heterogeneity was assessed by the Cochrane Q-test, using $\mathrm{I}^{2}$ statistic. Heterogeneity was considered significant as $\mathrm{P}<0.1$ or $\mathrm{I}^{2} \geq 50 \%$. Due to high heterogeneity in results, we also were done subgroup analysis based on molecular mechanisms, ethnicity and the expression level of lncRNAs in PC. The funnel plot asymmetry test as well as the Eggers' regression test were used to assess publication bias. Stata version 13.0 (StataCorp LP, College Station, TX, USA) was applied for the whole meta-analysis.

\section{Results}

As shown in Fig. 1, 336 articles were found in initial searches from PubMed, Web of Science and Scopus databases. After removing duplicate articles and screening by the title and abstracts, 191 full-text articles remained for further review. Of these, 152 studies were excluded due to insufficient data. Finally, a total of 39 studies which met our eligibility criteria were included in the current meta-analysis.

\section{Study characteristics}

A total of 39 eligible studies involving 4736 patients diagnosed with pancreatic cancer were included in this metaanalysis. These studies were published between 2014 and 2020. Of these, 38 studies were conducted in China [2054], and only one study in Turkey [55]. Samples were collected from tumor tissues in most studies, except three studies extracted them from blood [49], plasma [26] and serum [56]. All of these articles showed association of dysregulation of lncRNAs expression with different survival outcomes in PC. Overall survival (OS) [22-32, 3437, 39-49, 52-59], progression free survival (PFS) [39, 56], disease specific survival (DSS) [33], and disease free survival (DFS) [36] were investigated to evaluate survival outcomes. Expression of the lncRNAs was measured by use of quantitative real-time polymerase chain reaction (qRT-PCR) using GAPDH [21, 24, 26-29, 32-35, 37, 39, $40,42,43,48,50-53,55,57]$, $\beta$-actin [23, 29-31, 45-47], RNU6B [25, 38, 41, 44], U6 [20, 22, 56] and U7 as reference genes for endogenous normalization [51].
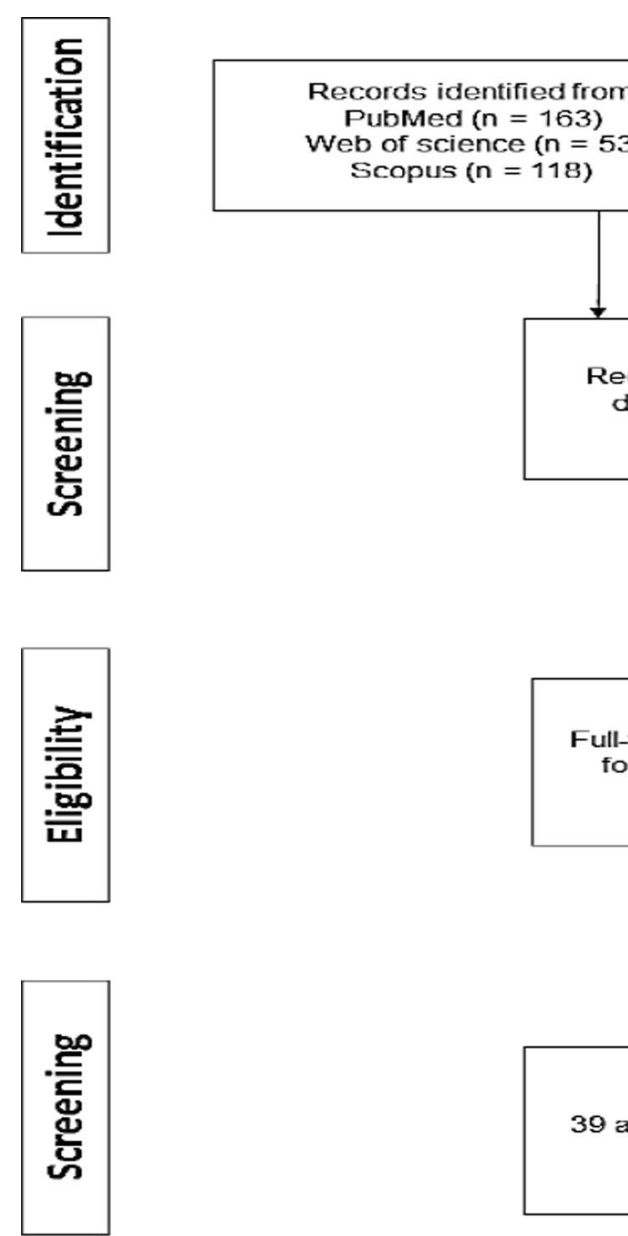
PubMed ( $\mathrm{n}=163)$ Scopus $(n=118)$

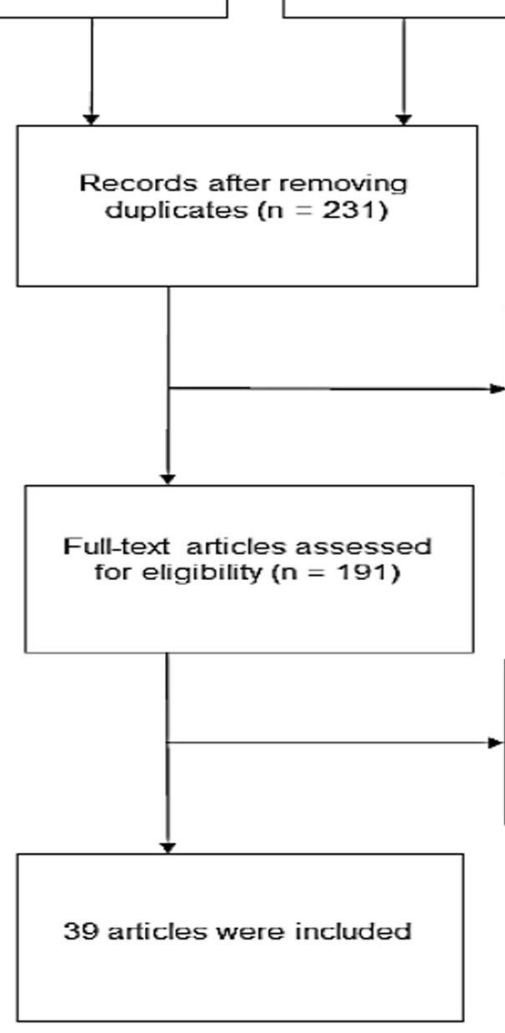

Additional records identified through other sources $(n=0)$

Records excluded by title and abstract (meta-analysis and review articles) $(n=40)$ with reasons $(n=152)$

Fig. 1 Study enrollment procedure in terms of the standards of the PRISMA diagram 
We assessed quality of included studies using the NOS tool. Given values ranged from 4 to 8 stars based of the number of parameter that analyzed in articles: 3 study was poor quality score and awarded 4 stars [40, 57, 58], 5 study achieved 6 stars and medium quality $[25,35,45,46$, 48], 13 studies gained 7 stars [22, 24, 30, 32, 36-38, 44, 50, 55, 59-61] and 18 articles awarded 8 stars with high quality [21, 23, 26-29, 31, 33, 34, 36, 39, 41, 43, 49, 51-53, $56,62]$. The characteristics of the included studies are summarized in Table 1.

\section{Association between IncRNAs expression and OS}

We conducted the present meta-analysis to figure out the value of aberrantly expressed lncRNAs in OS of 4691 PC patients from 39 studies. Statistical analyses represented significant association between the expression level of dysregulated lncRNAs and poor OS of PC patients in the relevant studies $(\mathrm{HR}=0.41,95 \% \mathrm{CI}$ $0.25 \pm 0.58, \quad \mathrm{I}^{2}=80.5 \%, \quad \mathrm{P}=0.000$, random-effects) as well as this effect in these studies analyzed by univariate analysis $\left(\mathrm{HR}=0.19,95 \% \mathrm{CI}-0.156 \pm 0.535, \mathrm{I}^{2}=0.0 \%\right.$ $\mathrm{P}=0.457)$ and multivariate analysis $(\mathrm{HR}=0.262,95 \% \mathrm{CI}$ $0.207 \pm 0.317, \mathrm{I}^{2}=81.7 \% \mathrm{P}=0.000$ ) (Fig. 2), while a significant heterogeneity existed between studies $\left(\mathrm{I}^{2}=80.5 \%\right.$, $\mathrm{P}=0.000$ ). Due to the presence of obvious heterogeneity, we performed subgroup analyses based on the ethnicity, molecular mechanisms and the expression level of lncRNAs in PC patients but similarly, heterogeneity was also assessed in our stratified analyses and there did not significant changes in heterogeneity after our subgrouping (Table 2).

\section{Association between IncRNAs expression and DFS}

The prognostic value of lncRNAs in DFS was explored in two studies including 260 patients. LncRNAs expression were significantly linked with DFS ( $\mathrm{HR}=0.51,95 \% \mathrm{CI}$ $0.19 \pm 0.83, P=0.00$, fixed-effects; Fig. 3 ), while no significant heterogeneity was observed in these studies.

\section{Correlation of IncRNAs with clinicopathological characteristics of pancreatic cancer}

Further stratified study grouped by clinicopathologic features exhibited that OS of patients with PC was markedly associated with gender (univariate analysis: $\mathrm{HR}=0.04$, $95 \% \mathrm{CI}-0.07$ to $0.16, \mathrm{P}=0.344$; multivariate analysis: $\mathrm{HR}=0.01,95 \% \mathrm{CI}-0.14$ to $0.17, \mathrm{P}=0.868$ ), Distance metastasis (univariate analysis: $\mathrm{HR}=0.02,95 \% \mathrm{CI}-0.53$ to $0.57, \mathrm{P}=0$; multivariate analysis: $\mathrm{HR}=0.08,95 \% \mathrm{CI}$ 0.02 to $0.13, \mathrm{P}=0.0$ ) Node metastasis (univariate analysis: $\mathrm{HR}=-0.12,95 \% \mathrm{CI}-0.34$ to $0.11, \mathrm{P}=0$; multivariate analysis: $\mathrm{HR}=0.20,95 \% \mathrm{CI} 0.12$ to $0.28, \mathrm{P}=0.0$ ) and other clinicopathologic factors demonstrated in Table 3.

\section{Publication bias}

The Funnel plot analysis was used to display asymmetry among the OS, DFS, distant metastasis, differentiation, gender, neural and prineural invasion, LNM, TNM and Stage (Fig. 4). Besides, no evidence of statistically significant publication bias observed by applying the Bgger tests and the Funnel plot analysis in combined prognostic studies.

\section{Sensitivity analysis}

Sensitivity analysis was performed to discover the influence of the individual study on the pooled results by removing one single study from the overall pooled analysis. The results depicted that no individual study significantly changed the pooled HRs (Fig. 5) demonstrating that our analysis was relatively stable and reliable. Also sensitivity analysis showed that no individual study had great influence on final results of our meta-analysis.

\section{Discussion}

Despite many advances in cancer research and treatment, the insidious onset of symptoms and extremely poor diagnosis of PC has still remained a controversial issue. The 5-year survival rate was estimated lower than 25\% resulting in worse clinical outcomes in PC [63]. Imaging methods including, computed tomography, magnetic resonance imaging and endoscopic ultrasound are currently available methods used in the diagnosis and prognosis of PC. Moreover, a number of serum biomarkers, such as circulating tumor DNA and certain microRNAs are used in these regard $[64,65]$. However, clinical application oh these methods in pancreatic cancer has been limited by their low specificity and sensitivity. Therefore, finding novel biomarkers are of most importance for early detection and more accurate treatment of this disease [66].

Over two past decades, numerous studies have focused on the potential roles of lncRNAs as contributors in various cell biological processes including gene and protein expression patterns. A growing body of evidence has verified the association between aberrant expressions of multiple lncRNAs with clinical outcomes for cancer patients. Notably, diagnostic significance of different lncRNAs profiling in digestive system tumors has been proved in numerous publications $[67,68]$. So, in order to investigate the promising prognostic biomarkers for $\mathrm{PC}$, as a high-degree malignancy of digestive system, the present systematic meta- analysis was performed to provide evidences to confirm potential association between altered lncRNAs and poor survival outcomes in PC. In this study, the information of $4736 \mathrm{PC}$ patients was extracted from 39 studies conducted between 2014-2020. Our results represented altered lncRNAs is significantly linked with 


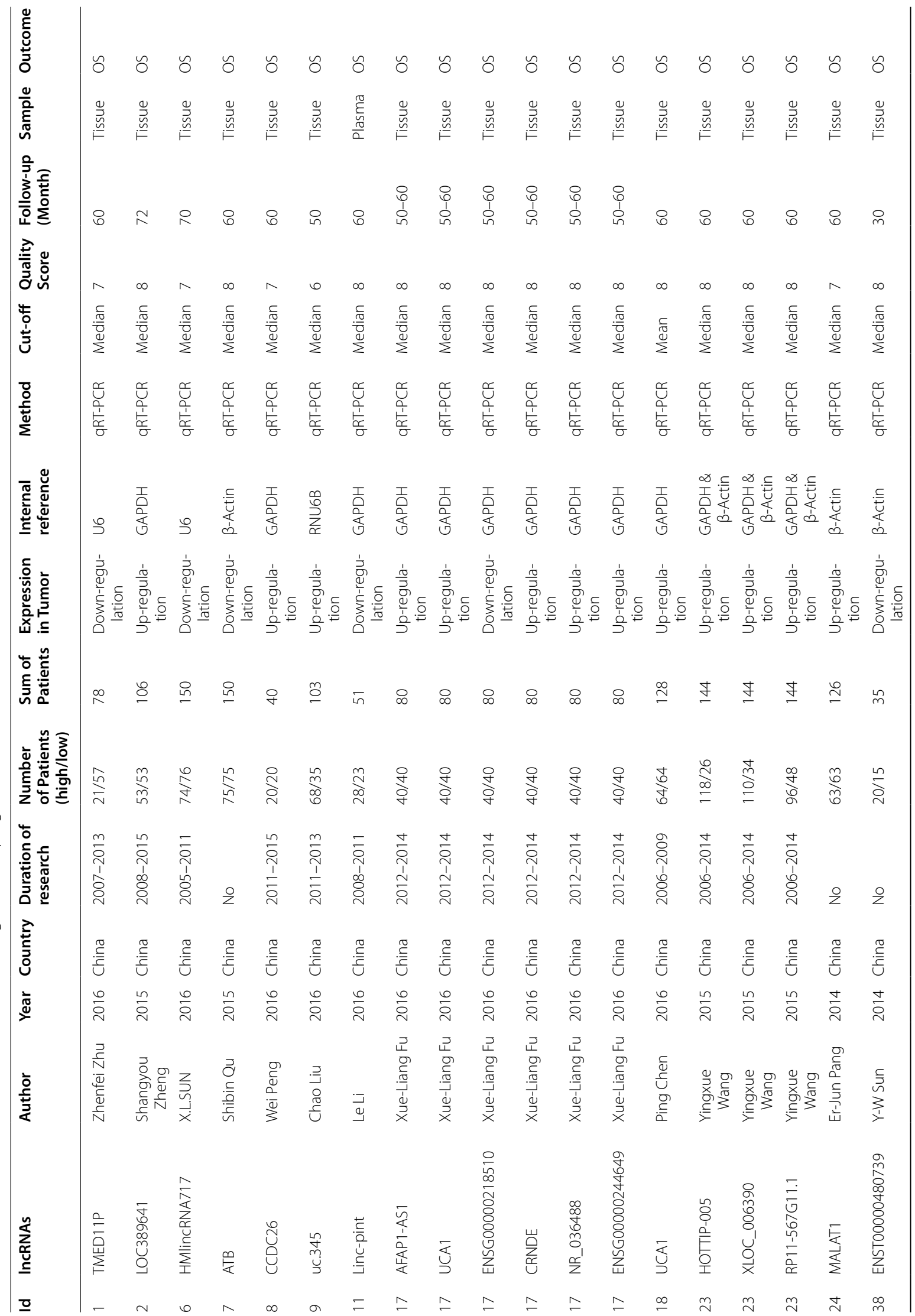




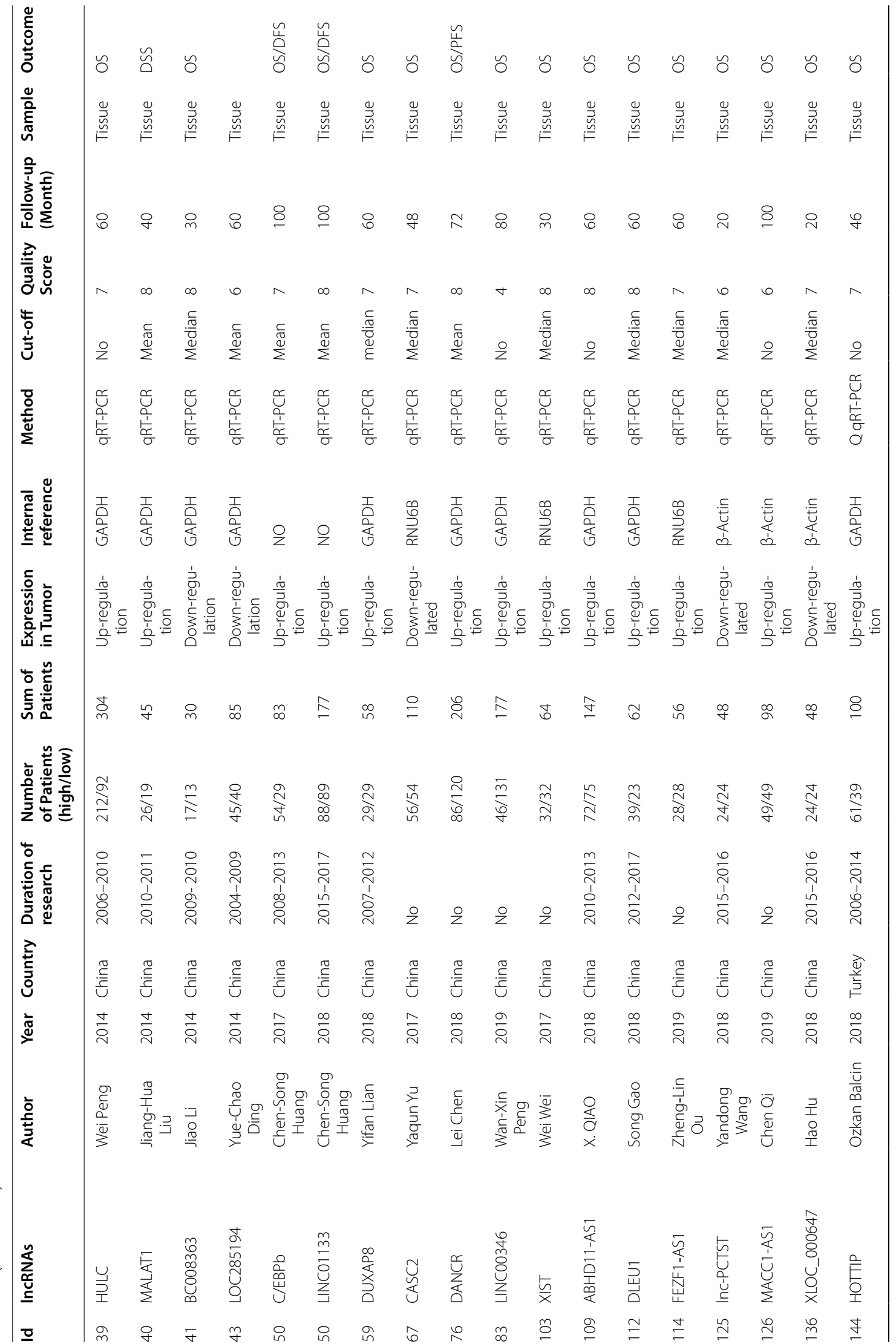




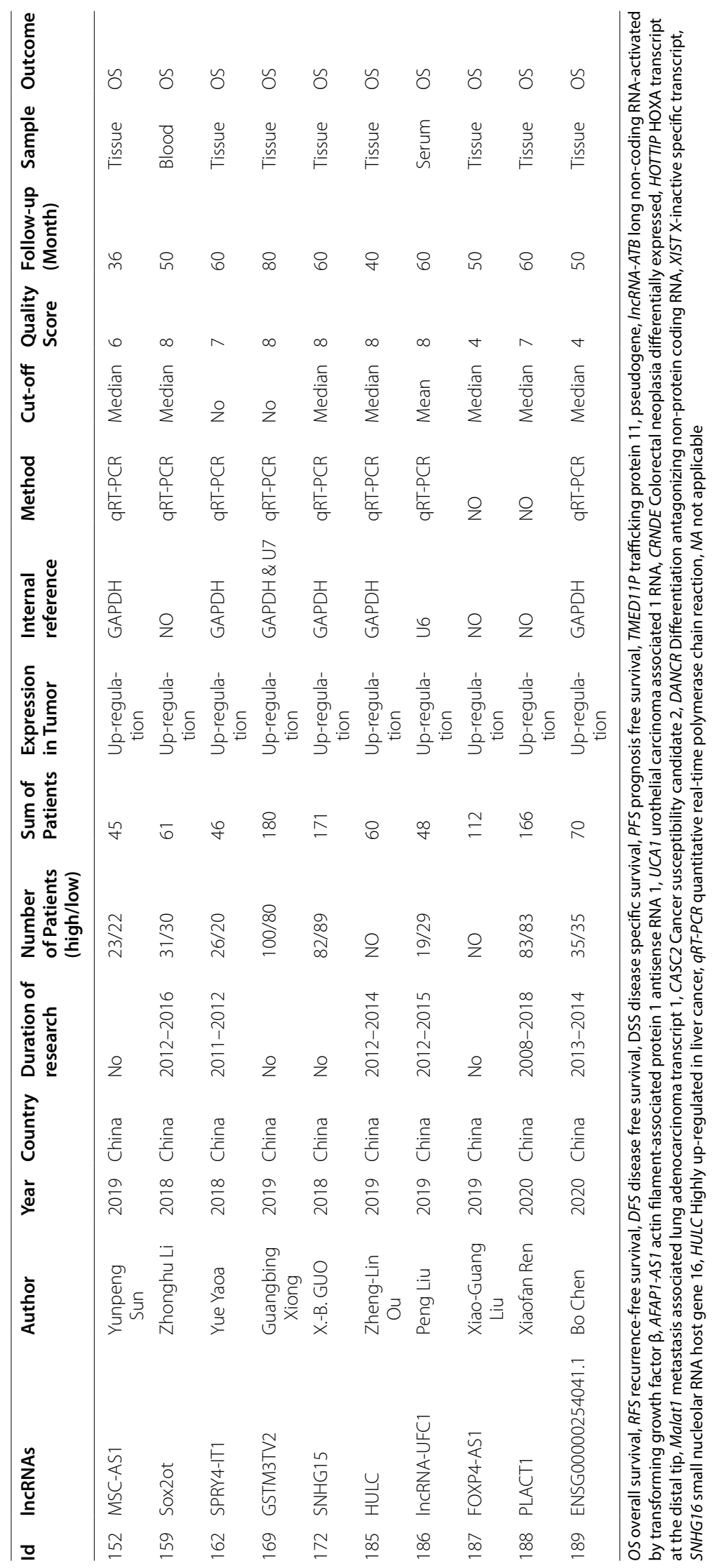




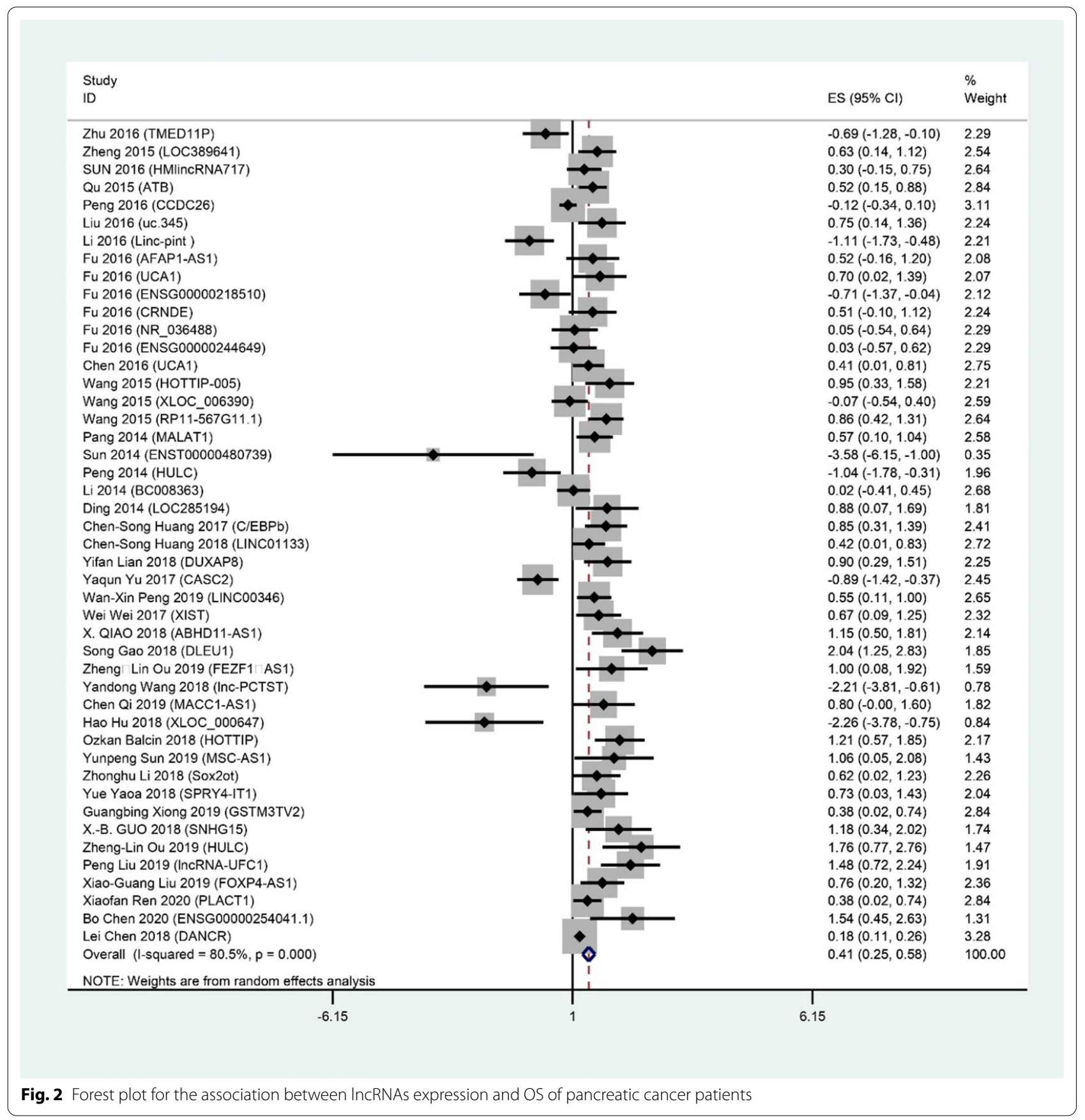

OS decline. Notably, we updated and augmented the reported results of meta-analysis carried out in 2017 with regard to the association between dysregulated lncRNAs and survival outcomes in PC [15].

In the current study, we assessed the prognostic role of different lncRNAs and their association with clinicopathological characteristics of PC. We found significant relation between altered expression of lncRNAs with poor OS period of $\mathrm{PC}(\mathrm{HR}=1.52$, with $95 \% \mathrm{CI} 1.04-2.22$, and $\mathrm{P}=0.031$ in univariate analysis; $\mathrm{HR}=1.55$, with $95 \%$ CI 1.19-2.02, and $P=0.001$ in multivariate analysis), suggesting that lncRNAs expression profile can be a prognostic biomarker of PC $[14,63,69]$.Correspondingly, our stratified analysis evidenced that the clinicopathological factors as Gender, distance metastasis, node metastasis, differentiation, neural and prineural invasion, vascular invasion, TNM, Stage were remarkably contributed with OS of PC. 
Table2 Main results of subgroup analyses

\begin{tabular}{|c|c|c|c|c|c|}
\hline Categories & Subgroups & $\mathbf{n}$ & $H R$ & $(95 \% \mathrm{Cl})$ & Heterogeneity \\
\hline All & & 46 & & & $80.5 \%$ \\
\hline \multirow[t]{2}{*}{ Ethnicity } & China & 45 & 0.25 & $(0.20,0.31)$ & $80.2 \%$ \\
\hline & Another country & 1 & 1.21 & $(0.57,1.85)$ & - \\
\hline \multirow[t]{2}{*}{ Expression level } & Up-regulation & 35 & 0.32 & $(0.27,0.38)$ & $73.6 \%$ \\
\hline & Down-regulation & 11 & -0.54 & $(-0.74,-0.34)$ & $71.9 \%$ \\
\hline \multirow[t]{6}{*}{ Molecular mechanisms } & Metastasis & 20 & 0.20 & $(0.14,0.26)$ & $80.7 \%$ \\
\hline & Proliferation & 31 & 0.23 & $(0.17,0.29)$ & $84.3 \%$ \\
\hline & Migration & 5 & 0.73 & $(0.50,0.96)$ & $74.0 \%$ \\
\hline & Invasion & 20 & 0.21 & $(0.09,0.33)$ & $84.2 \%$ \\
\hline & Tumorigenesis & 10 & 0.08 & $(-0.06,0.22)$ & $79.8 \%$ \\
\hline & Apoptosis & 11 & 0.27 & $(0.13,0.41)$ & $84.1 \%$ \\
\hline
\end{tabular}

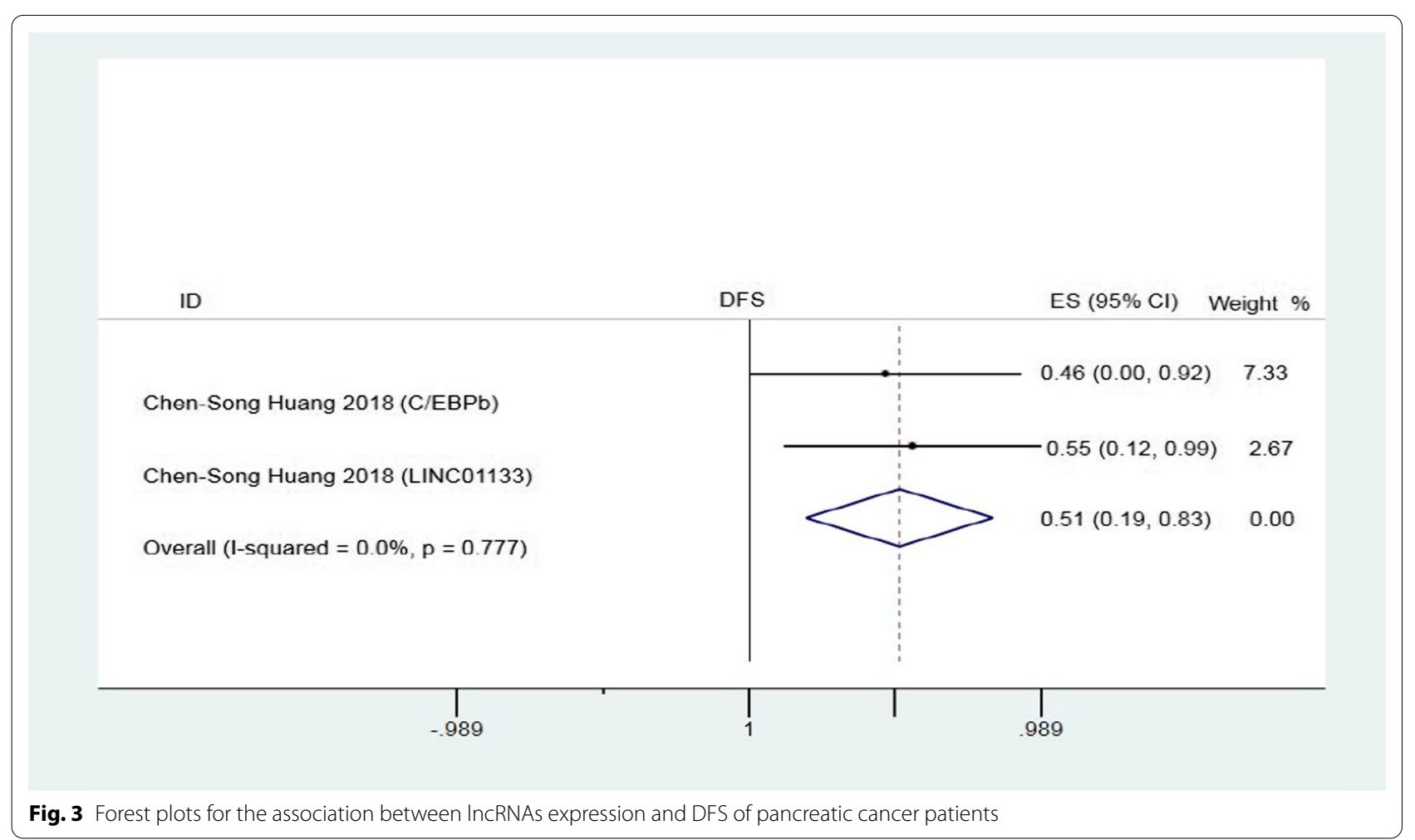

Moreover, large degree of heterogeneities among included studies were observed inspiring us to search its main causes from different aspects [70]. In this regard, we did subgroup analyses based on the ethnicity, molecular mechanisms and the expression level of lncRNAs in PC patients, however heterogeneity was also showed in our stratified analyses without any significant effect on heterogeneity.

Totally it could be concluded that lncRNAs expression profiling may serve as a helpful diagnostic and prognostic biomarker in of PC. So investigating the suitable single or panel of lncRNAs should be the focus of future studies [71-73].

However, it should be noted that there are several limitations in our meta-analysis including (1) The small sample sizes of the diagnostic meta-analysis as well as the limited clinical relevance of our results; (2) large heterogeneity in our analyses; (3) The HRs and 95\% CIs from some of articles could not be directly obtained and were estimated by software, which may decline the 


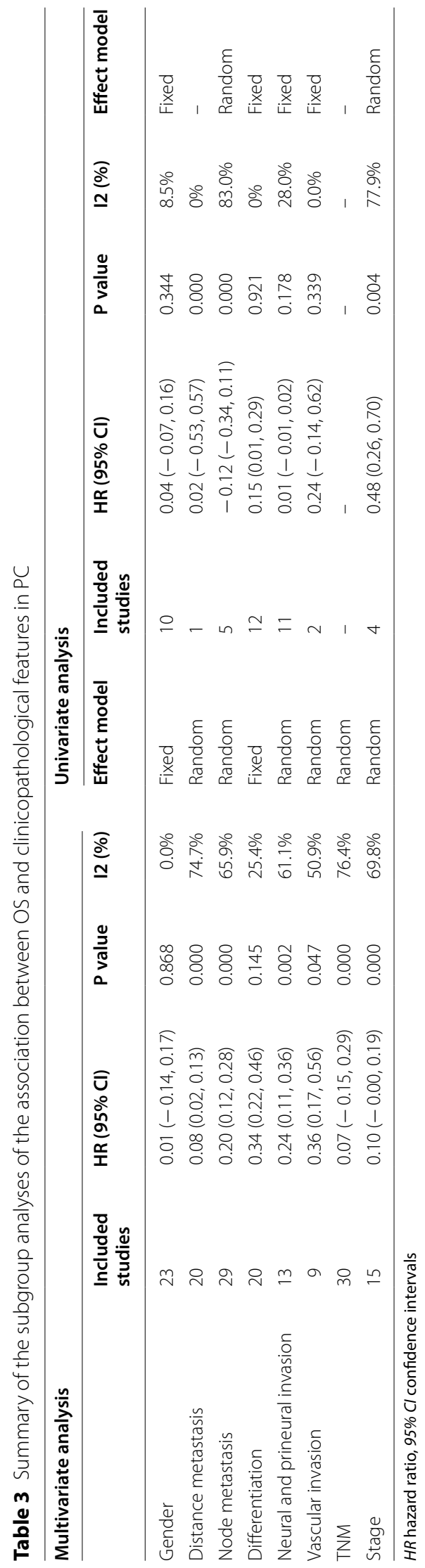




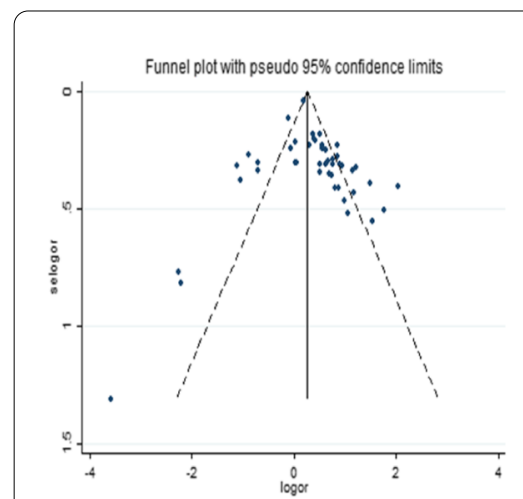

OS

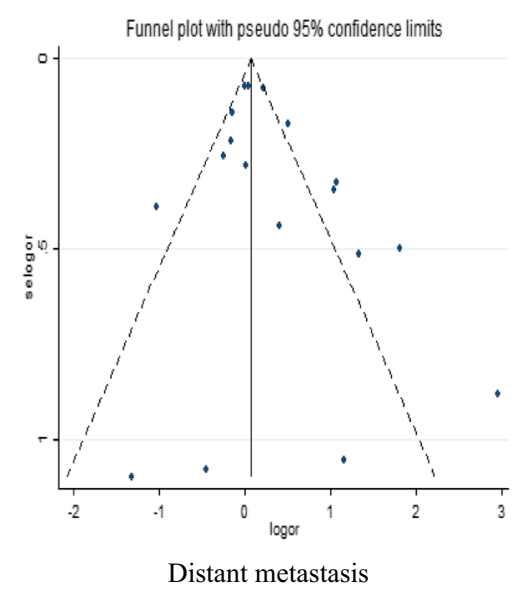

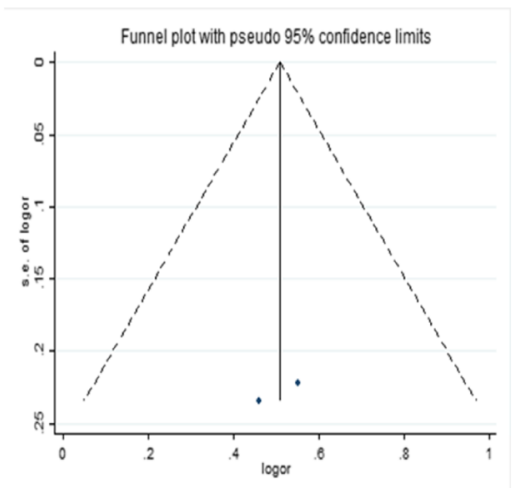

DFS

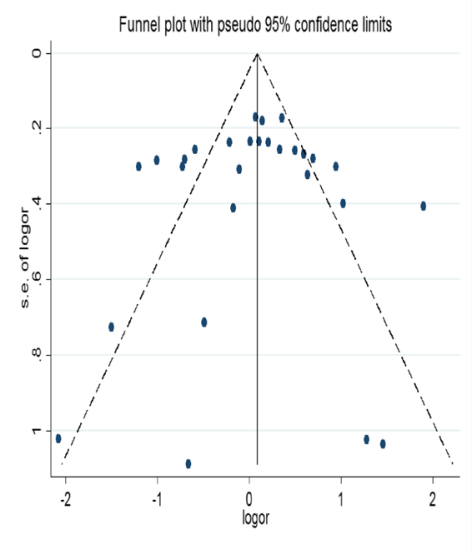

Gender invasion

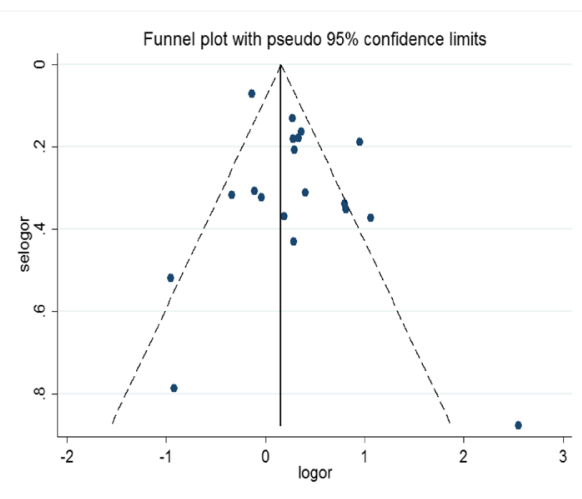

Stage

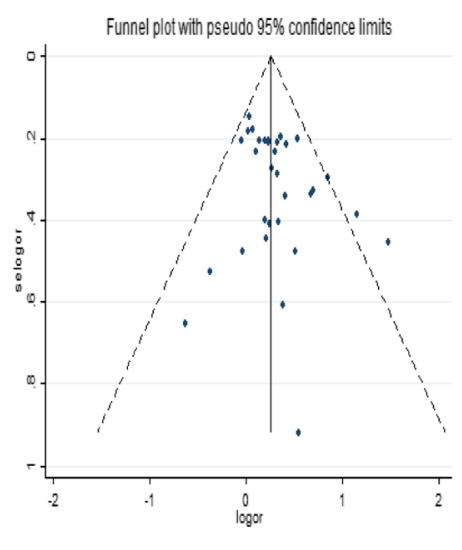

Neural and prineural

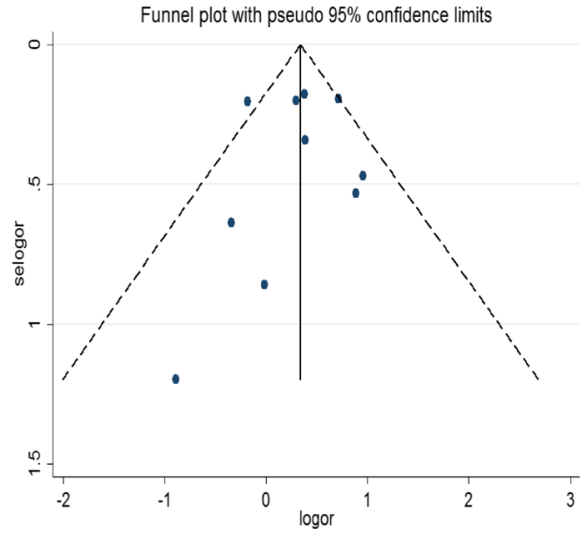

Vascular invasion

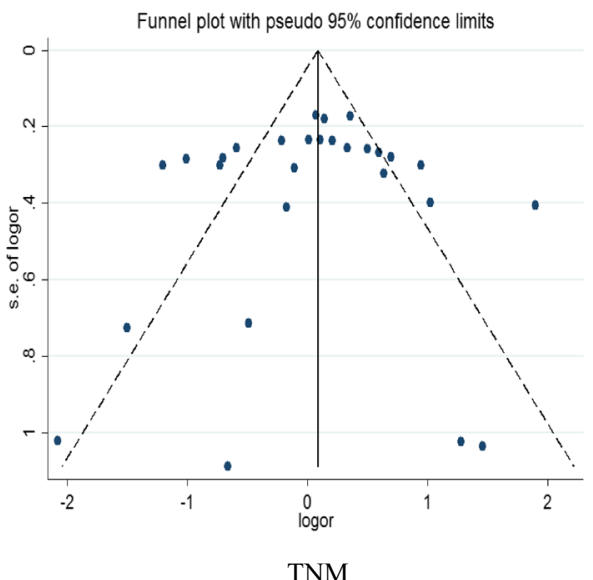

Fig. 4 Funnel plot analysis for publication bias

overall accuracy of the pooled effects. Totally, results from our study did not fully show the real clinical significance of lncRNA signature in PC, and in order to obtain a decisive conclusion, further comprehensive meta-analyses are needed to confirm the strong 


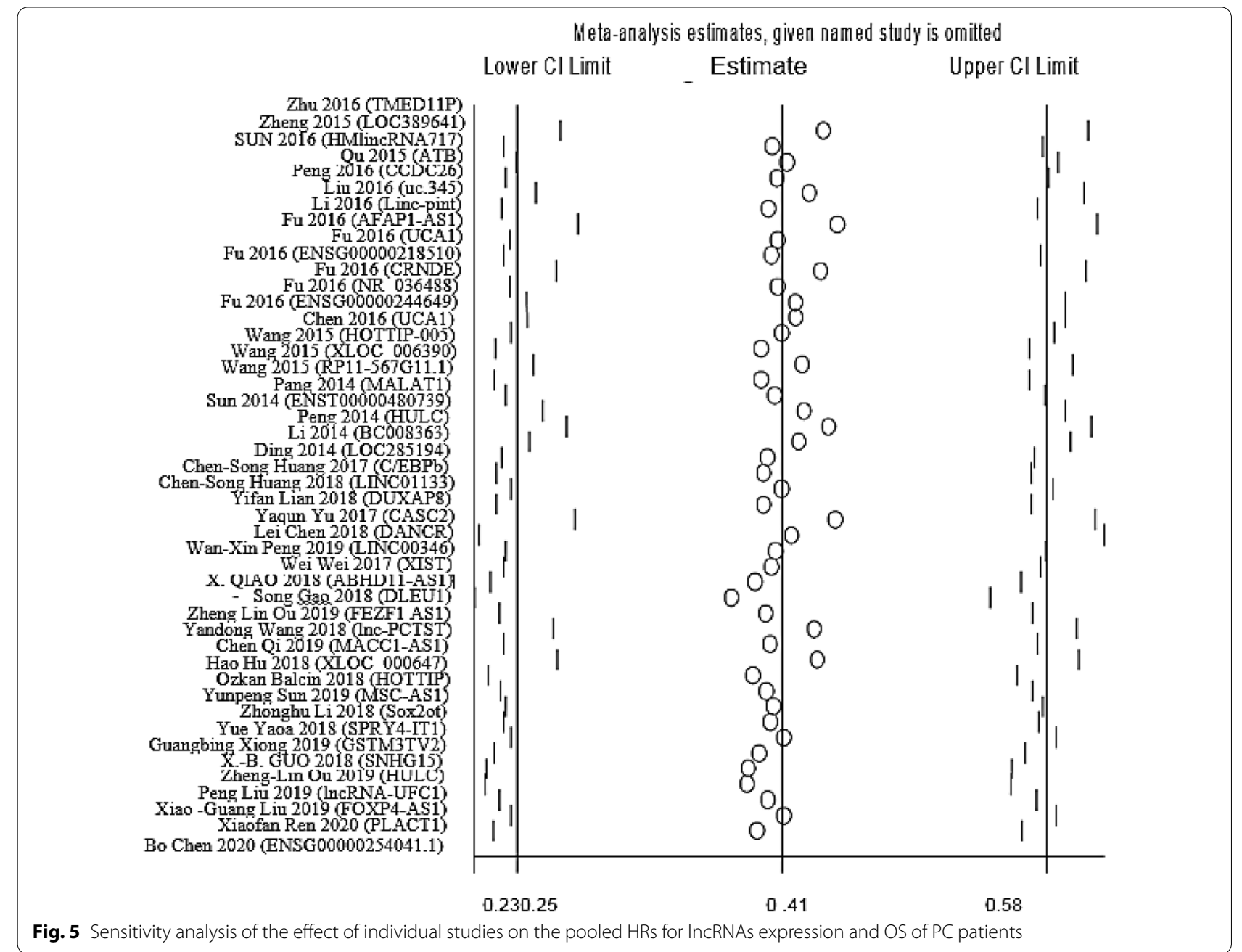

association between the expression pattern of lncRNAs and outcome of PC patients.

\section{Conclusion}

Altogether, our meta-analysis was updated and completed pervious reports to survey the prognostic value of lncRNAs and their association with clinical features of PC patients. Despite some above mentioned limitations, the present study revealed that IncRNAs could be used as potential prognostic markers for PC. However, more high quality and large-scale studies are still needed to validate the clinical utilities of lncRNAs in management of PC.

\section{Acknowledgements}

We would like to thank all our colleagues for their assistance. This study was supported by Research Deputy of Kashan University of Medical Sciences.

\section{Authors' contributions}

ESH, HHK and MAZ provided direction and guidance throughout the preparation of this manuscript. AS and AN conducted the literature and drafted the manuscript. $\mathrm{HN}$ and Fl reviewed the manuscript and made significant revisions on the drafts. All authors read and approved the final manuscript.

\section{Funding}

The financial support for the current research was provided by Research Deputy of Kashan University of Medical Sciences (Grant No. 96210), Kashan, Iran.

Availability of data and materials

Not applicable.

\section{Declarations}

Ethics approval and consent to participate

Not applicable.

\section{Consent for publication}

Not applicable.

Competing interests

The authors declared that they have no competing interests. 


\section{Author details}

${ }^{1}$ Gametogenesis Research Center, Kashan University of Medical Science, Kashan, Iran. ${ }^{2}$ Anatomical Sciences Research Center, Institute for Basic Sciences, Kashan University of Medical Sciences, Kashan, Iran. ${ }^{3}$ Student Research Committee, Kashan University of Medical Science, Kashan, Iran. ${ }^{4}$ Food and Drug Laboratory Research Center and Food and Drug Reference Control Laboratories Center, Food \& Drug Administration of Iran, MOH \& ME, Tehran, Iran.

Received: 8 December 2020 Accepted: 30 July 2021

Published online: 23 August 2021

\section{References}

1. King J, et al. Improving theranostics in pancreatic cancer. J Surg Oncol. 2017;116(1):104-13.

2. Harrow J, et al. GENCODE: the reference human genome annotation for the ENCODE Project. Genome Res. 2012;22(9):1760-74.

3. Hosseini ES, et al. Dysregulated expression of long noncoding RNAs in gynecologic cancers. Mol Cancer. 2017;16(1):1-13.

4. Yu WD, et al. Long noncoding RNAs in cancer-immunity cycle. J Cell Physiol. 2018;233(9):6518-23.

5. Hosseini ES, et al. Altered long non-coding RNAs expression and cytotoxic and anti-proliferative activity of dendrosomal nano-curcumin in ovarian cancer cells. Indian J Gynecol Oncol. 2021;19(2):1-9.

6. Huang $X$, et al. LncRNAs in pancreatic cancer. Oncotarget. 2016;7(35):57379.

7. Previdi MC, et al. Noncoding RNAs as novel biomarkers in pancreatic cancer: what do we know? Future Oncol. 2017;13(5):443-53.

8. Qu Z, et al. Meta-analysis of the prognostic value of abnormally expressed IncRNAs in hepatocellular carcinoma. Onco Targets Ther. 2016;9:5143.

9. Hosseini ES, et al. Studies on combination of oxaliplatin and dendrosomal nanocurcumin on proliferation, apoptosis induction, and long non-coding RNA expression in ovarian cancer cells. Cell Biol Toxicol. 2019;35(3):247-66.

10. Hosseini ES, et al. The role of altered long noncoding RNAs in overall survival of ovarian cancer: a systematic review and meta-analysis. Pathol Res Pract. 2021;219:153363.

11. Tian T, et al. The impact of IncRNA dysregulation on clinicopathology and survival of breast cancer: a systematic review and meta-analysis. Mol Ther Nucleic Acids. 2018;12:359-69.

12. Ma W, et al. The prognostic value of long noncoding RNAs in prostate cancer: a systematic review and meta-analysis. Oncotarget. 2017;8(34):57755.

13. Chi S, et al. Prognostic and diagnostic significance of IncRNAs expression in cervical cancer: a systematic review and meta-analysis. Oncotarget. 2017:8(45):79061.

14. Ning $L$, et al. Altered long noncoding RNAs and survival outcomes in ovarian cancer: a systematic review and meta-analysis (PRISMA Compliant). Medicine. 2018:97(32):e11481.

15. Lei $L$, et al. Meta-analysis of the clinical value of abnormally expressed long non-coding RNAs for pancreatic cancer. Oncotarget. 2017;8(51):89149.

16. Moher $D$, et al. Preferred reporting items for systematic reviews and meta-analyses: the PRISMA statement. PLoS Med. 2009;6(7):e1000097.

17. Fu W, et al. NFKB1-94insertion/deletion ATTG polymorphism and cancer risk: evidence from 50 case-control studies. Oncotarget. 2017;8(6):9806.

18. Stang A. Critical evaluation of the Newcastle-Ottawa scale for the assessment of the quality of nonrandomized studies in meta-analyses. Eur J Epidemiol. 2010;25(9):603-5.

19. Tierney JF, et al. Practical methods for incorporating summary time-toevent data into meta-analysis. Trials. 2007;8(1):16

20. Zhu Z, et al. Decreased expression of IncRNA TMED11P is correlated with progression and prognosis in pancreatic ductal adenocarcinoma. Int J Clin Exp Pathol. 2016;9(10):10550-6.

21. Zheng S, et al. Long non-coding RNA LOC389641 promotes progression of pancreatic ductal adenocarcinoma and increases cell invasion by regulating E-cadherin in a TNFRSF10A-related manner. Cancer Lett. 2016:371(2):354-65.
22. Sun $X$, et al. Association of LncRNA HMlincRNA717 with prognosis in pancreatic cancer. Eur Rev Med Pharmacol Sci. 2016;20(11):2230-4.

23. Qu S, et al. Downregulation of IncRNA-ATB correlates with clinical progression and unfavorable prognosis in pancreatic cancer. Tumor Biol. 2016;37(3):3933-8.

24. Peng W, Jiang A. Long noncoding RNA CCDC26 as a potential predictor biomarker contributes to tumorigenesis in pancreatic cancer. Biomed Pharmacother. 2016;83:712-7.

25. Liu C, et al. Long noncoding RNA uc. 345 promotes tumorigenesis of pancreatic cancer by upregulation of hnRNPL expression. Oncotarget. 2016;7(44):71556.

26. Le Li G-QZ, et al. Plasma and tumor levels of Linc-pint are diagnostic and prognostic biomarkers for pancreatic cancer. Oncotarget. 2016;7(44):71773

27. Fu X-L, et al. Analysis of long non-coding RNA expression profiles in pancreatic ductal adenocarcinoma. Sci Rep. 2016;6:33535.

28. Chen $\mathrm{P}$, et al. Long non-coding RNA UCA1 promotes the tumorigenesis in pancreatic cancer. Biomed Pharmacother. 2016;83:1220-6.

29. Wang Y, et al. Expression profile of long non-coding RNAs in pancreatic cancer and their clinical significance as biomarkers. Oncotarget 2015;6(34):35684

30. Pang E-J, et al. Overexpression of long non-coding RNA MALAT1 is correlated with clinical progression and unfavorable prognosis in pancreatic cancer. Tumor Biol. 2015;36(4):2403-7.

31. Sun Y, et al. A novel long non-coding RNA ENST00000480739 suppresses tumour cell invasion by regulating OS-9 and HIF-1a in pancreatic ductal adenocarcinoma. Br J Cancer. 2014;111(11):2131-41.

32. Peng W, Gao W, Feng J. Long noncoding RNA HULC is a novel biomarker of poor prognosis in patients with pancreatic cancer. Med Oncol. 2014;31(12):346.

33. Liu J-H, et al. Expression and prognostic significance of IncRNA MALAT1 in pancreatic cancer tissues. Asian Pac J Cancer Prev. 2014;15(7):2971-7.

34. Li J, et al. Long non-coding RNAs expressed in pancreatic ductal adenocarcinoma and IncRNA BC008363 an independent prognostic factor in PDAC. Pancreatology. 2014;14(5):385-90.

35. Ding Y-C, et al. Expression of long non-coding RNA LOC285194 and its prognostic significance in human pancreatic ductal adenocarcinoma. Int J Clin Exp Pathol. 2014;7(11):8065.

36. Huang C-S, et al. The C/EBPß-LINC01133 axis promotes cell proliferation in pancreatic ductal adenocarcinoma through upregulation of CCNG1. Cancer Lett. 2018;421:63-72.

37. Lian Y, et al. DUXAP8, a pseudogene derived IncRNA, promotes growth of pancreatic carcinoma cells by epigenetically silencing CDKN1A and KLF2. Cancer Commun. 2018;38(1):64.

38. Yu Y, et al. HNF1A/CASC2 regulates pancreatic cancer cell proliferation through PTEN/Akt signaling. J Cell Biochem. 2019;120(3):2816-27.

39. Chen $L$, et al. IncRNA differentiation antagonizing nonprotein coding RNA overexpression accelerates progression and indicates poor prognosis in pancreatic ductal adenocarcinoma. Onco Targets Ther. 2018;11:7955.

40. Peng W-X, et al. LINC00346 promotes pancreatic cancer progression through the CTCF-mediated Myc transcription. Oncogene. 2019;38(41):6770-80

41. Wei W, et al. LnCRNA XIST promotes pancreatic cancer proliferation through miR-133a/EGFR. J Cell Biochem. 2017;118(10):3349-58.

42. Qiao $X$, et al. Long noncoding RNA ABHD11-AS1 predicts the prognosis of pancreatic cancer patients and serves as a promoter by activating the PI3K-AKT pathway. Eur Rev Med Pharmacol Sci. 2018;22:8630-9.

43. Gao S, et al. Long noncoding RNA DLEU1 aggravates pancreatic ductal adenocarcinoma carcinogenesis via the miR-381/CXCR4 axis. J Cell Physiol. 2019;234(5):6746-57.

44. Ou ZL, et al. Long noncoding RNA FEZF1-AS1 predicts poor prognosis and modulates pancreatic cancer cell proliferation and invasion through miR-142/HIF-1 $a$ and miR-133a/EGFR upon hypoxia/normoxia. J Cell Physiol. 2019;234(9):15407-19.

45. Wang $Y$, et al. Long non-coding RNA Inc-PCTST predicts prognosis through inhibiting progression of pancreatic cancer by downregulation of TACC-3. Int J Cancer. 2018;143(12):3143-54. 
46. Qi C, et al. Long non-coding RNA MACC1-AS1 promoted pancreatic carcinoma progression through activation of PAX8/NOTCH1 signaling pathway. J Exp Clin Cancer Res. 2019;38(1):1-12.

47. Hu H, et al. Long non-coding RNA XLOC_000647 suppresses progression of pancreatic cancer and decreases epithelial-mesenchymal transition-induced cell invasion by down-regulating NLRP3. Mol Cancer 2018;17(1):18

48. Sun Y, et al. The role of IncRNA MSC-AS1/miR-29b-3p axis-mediated CDK14 modulation in pancreatic cancer proliferation and Gemcitabineinduced apoptosis. Cancer Biol Ther. 2019;20(6):729-39.

49. Li Z, et al. Tumor-derived exosomal Inc-Sox2ot promotes EMT and stemness by acting as a ceRNA in pancreatic ductal adenocarcinoma. Oncogene. 2018;37(28):3822-38.

50. Yao Y, et al. Upregulated long non-coding RNA SPRY4-IT1 predicts dismal prognosis for pancreatic ductal adenocarcinoma and regulates cell proliferation and apoptosis. Gene. 2018;659:52-8.

51. Xiong G, et al. Long noncoding RNA GSTM3TV2 upregulates LAT2 and OLR1 by competitively sponging let- 7 to promote gemcitabine resistance in pancreatic cancer. J Hematol Oncol. 2019;12(1):97.

52. Guo $X$, Yin H, Wang J. Evaluating the diagnostic and prognostic value of long non-coding RNA SNHG15 in pancreatic ductal adenocarcinoma. Eur Rev Med Pharmacol Sci. 2018;22(18):5892-8.

53. Ou Z-L, Luo Z, Lu Y-B. Long non-coding RNA HULC as a diagnostic and prognostic marker of pancreatic cancer. World J Gastroenterol. 2019;25(46):6728.

54. Vogelzang NJ, et al. Clinical cancer advances 2011: annual report on progress against cancer from the American Society of Clinical Oncology. J Clin Oncol. 2012;30:88-109.

55. Balcin $\mathrm{O}$, et al. Overexpression of the long noncoding RNA homeoboxA transcript at the distal tip predicts poor prognosis in a KRAS-independent manner in periampullary region tumors. Pancreas. 2018;47(2):213-20.

56. Liu P, et al. Serum IncRNA-UFC1 as a potential biomarker for diagnosis and prognosis of pancreatic cancer. Int J Clin Exp Pathol. 2019;12(11):4125.

57. Chen B, et al. The IncRNA ENSG00000254041.1 promotes cell invasiveness and associates with poor prognosis of pancreatic ductal adenocarcinoma. Aging (Albany NY). 2020;12(4):3647.

58. Liu XG, et al. Identify potential clinical significance of long noncoding RNA forkhead box P4 antisense RNA 1 in patients with early stage pancreatic ductal adenocarcinoma. Cancer Med. 2020;9(6):2062-76.

59. Ren $X$, et al. IncRNA-PLACT1 sustains activation of NF-kB pathway through a positive feedback loop with $1 \mathrm{KBa} / \mathrm{E} 2 \mathrm{~F} 1$ axis in pancreatic cancer. Mol Cancer. 2020;19(1):1-19.
60. Zhu Z, et al. Decreased expression of IncRNA TMED11P is correlated with progression and prognosis in pancreatic ductal adenocarcinoma. Int J Clin Exp Med. 2016;9(10):10550-6.

61. Hu H, et al. Long non-coding RNA XLOC_000647 suppresses progression of pancreatic cancer and decreases epithelial-mesenchymal transition-induced cell invasion by down-regulating NLRP3. Mol Cancer. 2018;17(1):1-14.

62. Qiao X, et al. Long noncoding RNA ABHD11-AS1 predicts the prognosis of pancreatic cancer patients and serves as a promoter by activating the PI3K-AKT pathway. Eur Rev Med Pharmacol Sci. 2018;22(24):8630-9.

63. Xia Y, et al. The prognostic significance of long noncoding RNAs in bladder cancer: a meta-analysis. PLoS ONE. 2018;13(6):e0198602.

64. Vila-Navarro $E$, et al. Novel circulating miRNA signatures for early detection of pancreatic neoplasia. Clin Transl Gastroenterol. 2019;10(4):e00029.

65. Stroese AJ, et al. Circulating microRNA-99 family as liquid biopsy marker in pancreatic adenocarcinoma. J Cancer Res Clin Oncol. 2018;144(12):2377-90.

66. Karandish F, Mallik S. Biomarkers and targeted therapy in pancreatic cancer. Biomark Cancer. 2016:8:BIC.S34414.

67. Dai M, et al. Meta-signature LnCRNAs serve as novel biomarkers for colorectal cancer: integrated bioinformatics analysis, experimental validation and diagnostic evaluation. Sci Rep. 2017;7(1):1-11.

68. Zheng C, et al. Long noncoding RNAs as novel serum biomarkers for the diagnosis of hepatocellular carcinoma: a systematic review and metaanalysis. Clin Transl Oncol. 2017;19(8):961-8.

69. Cui $X$, Jing $X$, Wu $X$. The prognostic value of long non coding RNAs in cervical cancer: a meta-analysis. Oncotarget. 2017;8(37):62470.

70. von Hippel PT. The heterogeneity statistic I 2 can be biased in small metaanalyses. BMC Med Res Methodol. 2015;15(1):35.

71. Zhuo Z-J, et al. Associations between IncRNA MEG3 polymorphisms and neuroblastoma risk in Chinese children. Aging (Albany NY). 2018;10(3):481.

72. LiY, et al. H19 gene polymorphisms and neuroblastoma susceptibility in Chinese children: a six-center case-control study. J Cancer. 2019;10(25):6358

73. Li S, et al. LINC00673 rs1 1655237 C> T and susceptibility to Wilms tumor: a five-center case-control study. J Gene Med. 2019;21(12):e3133.

\section{Publisher's Note}

Springer Nature remains neutral with regard to jurisdictional claims in published maps and institutional affiliations.
Ready to submit your research? Choose BMC and benefit from:

- fast, convenient online submission

- thorough peer review by experienced researchers in your field

- rapid publication on acceptance

- support for research data, including large and complex data types

- gold Open Access which fosters wider collaboration and increased citations

- maximum visibility for your research: over 100M website views per year

At BMC, research is always in progress.

Learn more biomedcentral.com/submissions 